\title{
Lesión cutánea por radiación en angioplastía coronaria compleja
}

\author{
José Pascual Salas ${ }^{1}$, Efraín Gaxiola ${ }^{1}$, Yara Itzel Tejeda ${ }^{2}$, Alan Méndez $^{2}$. \\ 1. Hospital Ángeles del Carmen, Unidad de Cardiología, Centro Especializado en Terapia Endovascular. \\ 2. Hospital Ángeles del Carmen, Facultad de Medicina, Universidad Autónoma de Guadalajara.
}

\section{Resumen:}

Las lesiones cutáneas por radiación (LCR) son una complicación infrecuente, con un estimado de 3.600 casos de lesiones mayores reportados en la actualidad. Presentamos un caso de lesión eritematosa mayor por radiación posterior a angioplastía coronaria fallida y en segundo tiempo angioplastía coronaria con rotablación.

Palabras clave: radiación; lesión cutánea; angioplastía coronaria percutánea.

\section{Cutaneous radiation injury after failed percutaneous coronary angioplasty}

Cutaneous radiation injuries are an infrequent complication, with an estimated 3.600 cases of major injuries reported up to now. We present a case of a major erythematous lesion induced by radiation after failed coronary angioplasty and consecutive coronary rotablation.

Keywords: radiation; cutaneous injury; percutaneous coronary angioplasty.

\section{Correspondencia:}

José Pascual Salas Llamas

Cardiología Clínica e Intervencionista, DGP: 3736698

Hospital Ángeles del Carmen

doc.salas@hotmail.com 


\section{Introducción:}

Las lesiones cutáneas por radiación (LCR) son infrecuentes, pero son una seria complicación en procedimientos intervencionistas guiados por fluoroscopia. Tanto el paciente y el equipo médico están expuestos a este riesgo, con un riesgo mínimo pero definitivo. Estas lesiones comúnmente son poco reconocidas o mal diagnosticadas ${ }^{1}$. Para el paciente, la dosis efectiva de radiación para el diagnóstico coronario angiográfico y colocación de stent es de, aproximadamente, $5 \mathrm{mSv}$, y para angiogramas por tomografía computarizada y escáneres nucleares varía de $10 \mathrm{a} 25 \mathrm{mSv}$ para protocolos estándar ${ }^{2}$. La dosis de radiación cutánea incrementa con mayor fluoroscopía y mayor peso corporal del paciente ${ }^{3}$. Una exposición focal excesiva a los rayos-x produce lesiones dosis dependientes, con desarrollo de eritemas retardados con dosis absorbida de 6 Gy en un área localizada de la piel, y úlceras profundas necróticas en dosis en exceso de 18 Gy. Usualmente, estas lesiones ocurren en intervenciones percutáneas coronarias de larga duración y complicadas o procedimientos de ablación electrofisiológicos ${ }^{2}$.

El tratamiento base para LCR menores incluyen control del dolor y la prevención de infección, con una completa recuperación esperada de 3 a 6 meses posterior a la exposición. En lesiones más severas, el área afectada puede requerir trasplante cutáneo.

\section{Caso clínico:}

Paciente masculino de 48 años, originario de México, con antecedente de diabetes mellitus tipo 2, dislipidemia mixta, obesidad con peso $125 \mathrm{~kg}$, IMC $43.25 \mathrm{~kg} / \mathrm{m} 2$, en clase funcional NYHA III. Ingresó a urgencias por presentar disnea progresiva de medianos a pequeños esfuerzos y angina de pecho típica de isquemia, EKG con bloqueo de rama izquierda (BRIHH) completo, QRS de $180 \mathrm{~ms}$, Ecocardiograma transtoráxico con hipocinesia severa en región anterolateral, septal y lateral, FEVI 12\%, Eco-Dobutamina con mejoría de la contractilidad en segmento anterior a dosis máximas con incremento de FEVI > $10 \%$, positivo para isquemia. El cateterismo reveló una lesión severa suboclusiva de $95 \%$ en arteria descendente anterior (DA) segmento proximal, severamente calcificada. Se procedió a realizar angioplastía coronaria transluminal percutánea (ACTP) de DA sin éxito; sin lograr el cruce de balón o stent en el sitio de la lesión debido al abundante calcio. Se intentó con apoyo de Guideliner, Microcateter, Cutting Balloon 2.0 x $10 \mathrm{~mm}$ y 2.5 x 10 $\mathrm{mm}$ sin éxito con una duración de procedimiento aproximada de 3 horas, la dosis total de radiación fue de 11.5
Gy con fluoroscopio Artis Zee Floor Siemens. Tres días después se decidió realizar un segundo intento de ACTP con rotablación direccional, con Fresa Rotalink de inicio $1.25 \mathrm{~mm}$ y posteriormente $1.50 \mathrm{~mm}$, logrando el cruce de la de la lesión con rotablator. Posterior se realizó predilatación con balón de 2.0 x 18 mm y 2.5 x $18 \mathrm{~mm}$, y finalmente implante de stent medicado a DA de 3.0 x 30 $\mathrm{mm}$. Con apoyo de imagen Clear Stent se observó falta de expansión de stent en su segmento proximal por lo que se post dilató dicho segmento con balón NC $3.5 \mathrm{x}$ $12 \mathrm{~mm}$, con adecuada posición e implantación de este. Con una duración de procedimiento de 2 horas, dosis total de radiación de 13.47 Gy con fluoroscopio Artis Zee Floor Siemens. Mejoró la función sistólica con aumento de FEVI a $28 \%$ y mejoría de la clase funcional NYHA II, persistió el bloqueo de rama izquierda completo con QRS de $150 \mathrm{~ms}$, por lo que en un futuro inmediato se valorarán criterios de resincronización cardíaca.

A los 10 días del procedimiento, el paciente desarrolló una lesión eritematosa mayor en tórax posterior izquierdo, sin presentar síntomas de dolor o prurito. Se observó placa eritematosa de $14 \mathrm{~cm}$ de diámetro, bien definida de márgenes irregulares, conformada por múltiples pápulas y vesículas, con aumento de la temperatura local. Se interconsulta con los servicios de oncología y dermatología, con manejo a base de aseo diario con antisépticos locales, más aplicación de hidrocortisona tópica 2 veces al día durante 15 días y crema de neomicina y retinol por la noche durante 2 meses. Al mes de evolución con engrosamiento de la lesión, presentaba pigmentación e hiperqueratosis. A los 3 meses de seguimiento con hiperqueratosis, engrosamiento e hiperpigmentación cutánea, sin datos de inflamación local. A los 5 meses de seguimiento hubo disminución de pigmentación cutánea. La evolución a 8 meses continúa con mejoría, con disminución de la pigmentación cutánea y con mayor uniformidad de la lesión (Figura 1).

\section{Discusión:}

La LCR es una complicación rara que se asocia a inflamación del tejido que se manifiesta con dermatitis, necrosis y ulceración ${ }^{4}$. La liberación de factores secretores por células inflamatorias, mesenquimales y epiteliales que inducen fibroblastos, llevan a fibrosis que se demuestra con cambios en la textura de la piel, retracción, molestia, telangiectasia, dolor y prurito 5 .

En los últimos 30 años el número de intervenciones guiadas por fluoroscopía ha aumentado drásticamente, con números duplicados cada 2-4 años en algunos paí- 

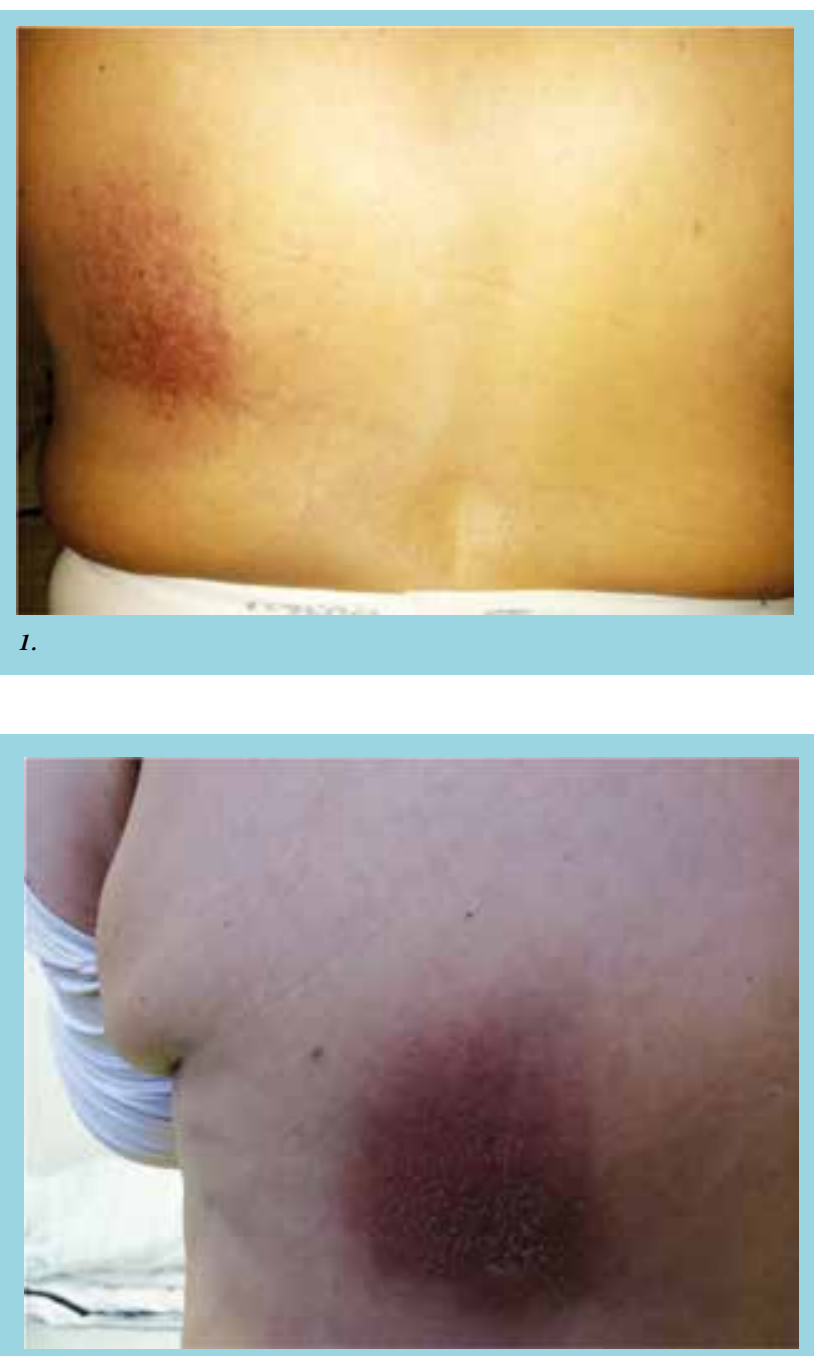

2.

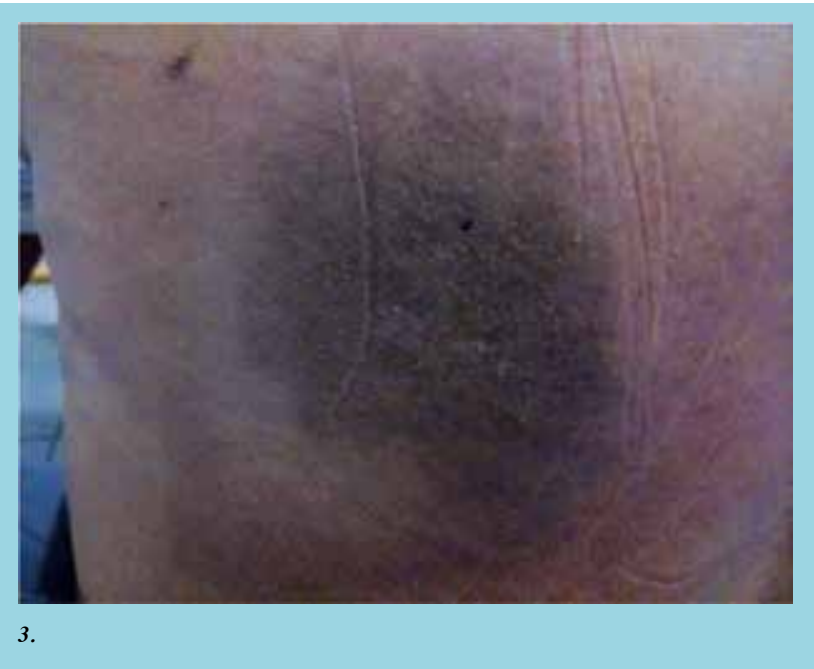

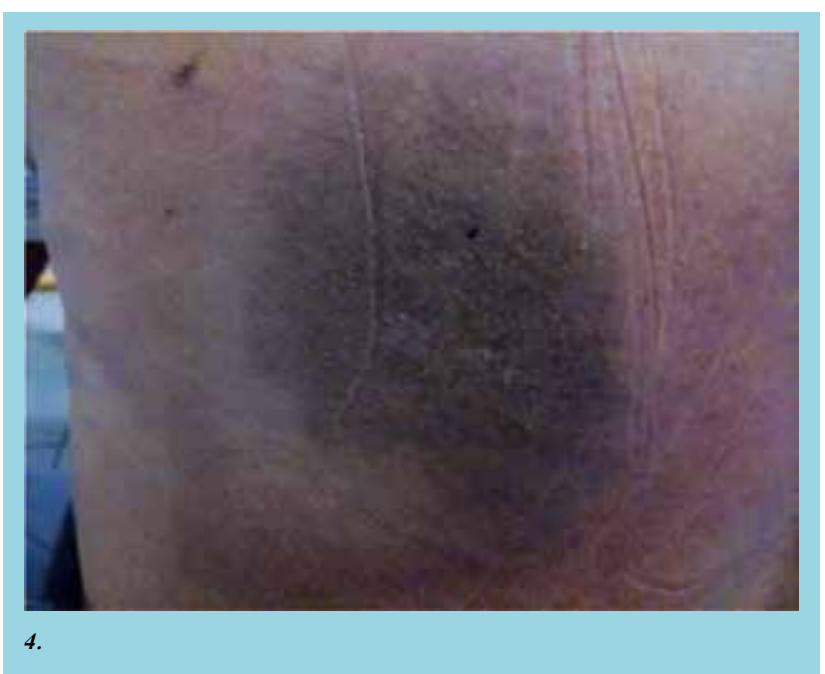

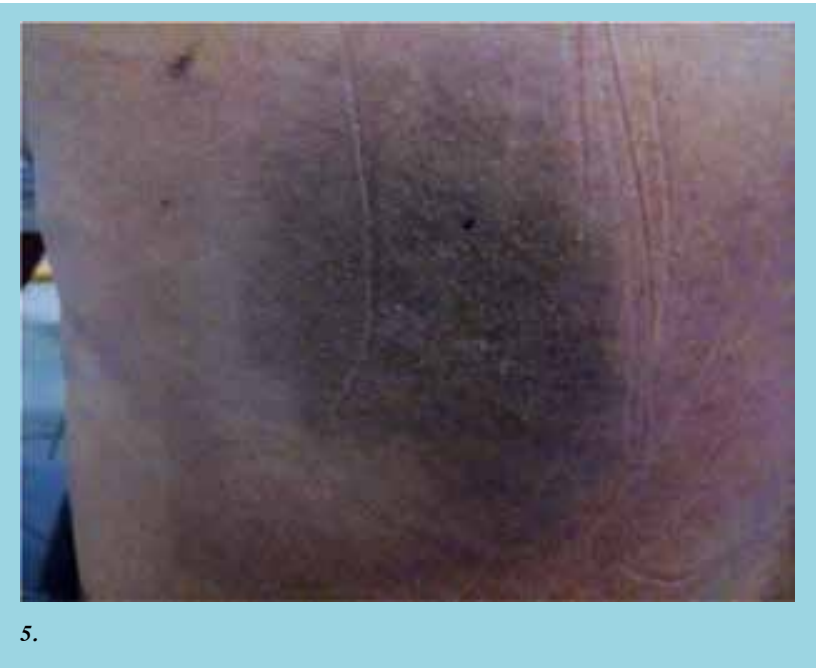

ses $^{6}$. Con este creciente número, la incidencia de LCR ha aumentado, estimada actualmente entre 1:10.000 a 1:100.000 procedimientos, pero el riesgo verdadero se desconoce ${ }^{4}$.

En nuestro caso, con una dosis total de 24.97 Gy en dos procedimientos la gran acumulación de radiación en el paciente llevó en poco tiempo al desarrollo de LCR. Se detectó inmediatamente la lesión y se relacionó a la exposición del paciente a la radiación ya que apareció en región del tórax posterior izquierdo, sobre la cual recayó la prolongada irradiación.

Es escasa la literatura de LCR, con un estimado de 3.600 casos de lesiones mayores reportados en la actualidad 4 . En una serie de 14 casos analizados (Vano 2010) sometidos a angiografía coronaria y ACTP 3 pacientes desarrollaron lesiones cutáneas. Un caso con dosis estimada de 
9.5 Gy presentó leve lesión cutánea, otro caso con dosis estimada de 7.3 Gy telangiectasia y pigmentación discreta, y en otro paciente con lupus eritematoso cutáneo con pigmentación del área radiada en dosis de $5.6 \mathrm{~Gy}^{7}$. Koenig et al, reportó 38 casos de necrosis y ulceración crónica en 73 pacientes sometidos a intervención guiada por fluoroscopía. Catorce de ellos requirieron trasplante cutáneo $^{8}$. Se han reportado casos hasta con dosis cutáneas que exceden $60 \mathrm{~Gy} 9$.

Además de representar un riesgo para el paciente, en los últimos dos años se reportan más casos de daños por radiación al operador, entre los cuales destacan afecciones cerebrales, oculares y tiroideas. Las primeras, concernientes respecto a tumores cerebrales surgieron en 1998 al reportarse 2 casos de tumor cerebral en cardiólogos de Canadá ${ }^{10}$. En Suecia, se identificaron 3 casos más ${ }^{11}$. Más aun, se ha observado un predominio de las lesiones tumorales cerebrales en el hemisferio izquierdo, asociado a una mayor exposición de radiación del lado izquierdo que el derecho ${ }^{12,13}$. Otra complicación asociada es el desarrollo de cataratas con mayor afectación del lente subcapsular posterior ${ }^{14,15}$. Por último, la exposición a la radiación se ha asociado al desarrollo de enfermedades estructurales tiroideas (tumores, nódulos) y funcio- nales (hiper o hipotiroidismo) observadas en diferentes series ${ }^{16}$.

Se ha observado que la mejor prevención de la lesión por radiación para el paciente y el equipo médico es minimizar el tiempo total de fluoroscopía, mantener el intensificador de imagen cerca del paciente, dirigir hacia la región de interés, maximizar la distancia del operador con el paciente, usar un apropiado equipo de protección y monitorización de la dosis de radiación ${ }^{4}$.

\section{Conclusión:}

Las LCR son una complicación infrecuente, que se puede presentar en procedimientos de larga duración como en ACTP compleja, o en casos de oclusión crónica total. Por ello, es importante tomar en cuenta las medidas de prevención necesarias para reducir riesgo de LCR, tanto del paciente como lesiones por radiación del equipo médico. En diferentes centros de intervencionismo del mundo se emplean actualmente terapias en intervencionismo estructural y el tiempo de radiación es mayor. Siguen existiendo, hay centros en los que no se toman las medidas necesarias de protección radiológica, tanto para el paciente como para el personal ocupacionalmente expuesto. 


\section{Referencias:}

1. SLOVUT DP. Cutaneous Radiation Injury After Complex Coronary Intervention. JACC Cardiovascular Interventions. 2009;2:2

2. VILESTRA RE, WAGNER LK. X-ray Burns-Painful, Protracted and Preventable. Clinical Cardiology. 2008;31:4

3. HWANG E, GAXIOLA E, VLIETSTRA RE, BRENNER A, EBERSOLE D, BROWNE K. Real-time measurement of skin radiation during cardiac catheterization. Catheterization and Cardiovascular Diagnosis. 1998;43:367-70

4. AKITA S. Treatment of Radiation Injury. Advances in Wound Care. 2014;3:1

5. COX JD, STETZ J, PAJAK TF. Toxicity criteria of the radiation therapy oncology group (RTOG) and the European organization for research and treatment of cancer (EORTC). International Journal of Radiation Oncology Biology Physics. $1995 ; 31: 1341$

6. JASCHKE W, SCHMUTH M, TRIANNI A, BARTAL G. Radiation-Induced Skin injuries to Patients: What the Interventional Radiologist Need to Know. Cardiovascular and Interventional Radiology. 2017;40:1131-1140

7. VANO E, GOICOLEA J, GALVAN C, GONZALEZ L, MEIGGS L, TEN JI, MACAYA C. Skin radiation injuries in patients following repeated coronary angioplasty procedures. British Journal of Radiology. 2014;74:1023-1031

8. KOENIG TR, WOLFF D, METTLER FA, WAGNER LK. Skin Injuries from Fluoroscopically Guided Procedures: Part I, Characteristics of Radiation Injury. American Journal of Roentgenology. 2001; 177: 3-11
9. METTLER FA JR, KOENIG TR, WAGNER LK, KELSEY CA. Radiation injuries after fluoroscopic prodecures. Seminars in ULTRASOUND CT and MRI. 2002;23:428-42

10. FINKELSTEIN MM. Is brain cancer an occupational disease of cardiologists? Canadian Journal of Cardiology. $1998 ; 14: 1385-8$

11. HARDELL L, MILD KH, PÅHLSON A, HALLQUIST A. Ionizing radiation, cellular telephones and the risk for brain tumours. European Jurnal of Cancer Prevention. 2001;10:523-9

12. ROQUIN A, GOLDSTEIN J, BAR O. Brain tumours among interventional cardiologists: a cause for alarm? Report of four new cases from two cities and a review of the literature. Eurointervention. 2012;7:1081-6

13. ROGUIN A, GOLDSTEIN J, BAR O. Brain and neck tumors among physicians performing interventional procedures. American Journal of Cardiology. 2013;111:1368-72

14. JACOB S, BOVEDA S, BAR O, et al. Interventional cardiologists and risk of radiation-induced cataract: results of a French multicenter observational study. International Journal of Cardiology. 2013;167:1843-7

15. VANO E, KLEIMAN NJ, DURAN A, et al. Radiation-associated lens opacities in catheterization personnel: results of a survey and direct assessments. Journal of Vascular and Interventional Radiology. 2013;24:197-204

16. RON E, BRENNER A. Non-malignant thyroid diseases after a wide range of radiation exposures. Radiation Research. 2010;174:877-88 